\title{
La formación de liderazgo en tiempos de gerenciamiento. La perspectiva de estudiantes en una escuela de elite del norte del conurbano
} bonaerense

\section{Leadership training in times of management. Students' perspective in an elite school of the northern outskirts of the city of Buenos Aires}

Di Nucci, Leandro; Langer, Eduardo

Leandro Di Nucci Sobre el autor

leandinucci@gmail.com

Universidad Nacional de San Martín (UNSAM), Argentina

Eduardo Langer Sobre el autor langereduardo@gmail.com Consejo Nacional Científico y Técnico (CONICET), Argentina

\section{Itinerarios educativos}

Universidad Nacional del Litoral, Argentina

ISSN: $1850-3853$

ISSN-e: 2362-5554

Periodicidad: Anual

vol. 1, núm. 14, 2021

revistadelindi@fhuc.unl.edu.ar

Recepción: 13 Noviembre 2020

Aprobación: 27 Junio 2021

URL: https://doi.org/10.14409/ie.2021.14.e0002

\section{c) (i) (2)}

Esta obra está bajo una Licencia Creative Commons AtribuciónNoComercial-CompartirIgual 4.0 Internacional.
Resumen: El artículo aborda la descripción de las prácticas en una escuela de elite y sus relaciones con las elecciones futuras de jóvenes de sectores altos para comprender los procesos de fragmentación y desigualdad educativa desde los grupos mejor posicionados socialmente. La interrogación gira en torno a las particularidades que presentan los dispositivos pedagógicos en las sociedades de gerenciamiento atendiendo a los discursos de la formación en liderazgo y sus correspondencias con los sentidos laborales que asignan los estudiantes. Para ello se retoman resultados de investigación que abarcaron técnicas cualitativas múltiples a partir del estudio en caso en una escuela del conurbano bonaerense.

Palabras clave: formación, liderazgo , gerenciamiento , estudiantes, escuela secundaria.

Abstract: This article addresses usual practices in an elite secondary school and their correspondence with the future choices of young people from high-income sectors in order to understand the processes of educational fragmentation and inequality from the point of view of the best socially positioned groups. The question revolves around the particularities posed by pedagogical devices within societies of managing, taking into account the discourse of leadership training and its correspondence with the meanings of work conveyed by students. For this purpose, the article includes research results from the use of multiple qualitative techniques applied to the case study of a school in the outskirts of the city of Buenos Aires.

Keywords: training, leadership, management, students, secondary school.

\section{Introducción}

Hace ya algunas décadas, los sectores altos de la población «optan» por segregarse del resto de la sociedad como un modo de cercar espacios y territorios exclusivos. De lo tratan es de separarse, distinguirse, auto-segregarse, perder 
contacto con el afuera «peligroso», elegir dónde vivir o estudiar como algunas de las dimensiones centrales desde las cuáles se puede indagar estos procesos en la población de elite.

En este contexto, se producen discursos y prácticas en las escolaridades, como advierten Tiramonti y Ziegler (2008), propias del mundo empresarial, donde se resalta la figura del individuo como libre y responsable de sí mismo (Langer, 2017), se busca formar sujetos «aptos», competitivos y exitosos capaces de triunfar gracias a su propia formación en la era del gerenciamiento (Grinberg, 2008). Esas escolaridades consideran a sus alumnos como los futuros líderes de la sociedad y son las familias junto a sus hijos que llegan allí en busca de ese objetivo ya que, tal como describiremos más adelante, se ven y piensan a sí mismos ocupando cargos gerenciales o políticos en sus vidas laborales.

En este sentido, el artículo aborda la descripción de las prácticas pedagógicas en una escuela de elite y las perspectivas sobre las posibles elecciones futuras de jóvenes de sectores altos. Conocer acerca de los sentidos en estos sectores, siguiendo a Tiramonti y Ziegler (2008), «resulta fundamental para comprender los procesos de fragmentación y desigualdad educativa, ya que permite percibir las distancias y diferencias tanto entre los grupos mejor posicionados socialmente, como entre estos y los sectores que poseen más desventajas comparativas» (65). Es decir, estudiar el funcionamiento de una escuela ubicada territorialmente en el norte del conurbano bonaerense a la que asisten alumnos pertenecientes a los sectores de privilegio, permite delimitar no solo la producción de un determinado dispositivo pedagógico (Grinberg, 2008; Langer, 2017; Armella, 2016) en escuelas de sectores de elite que aspiran a la formación de y en liderazgos sino a describir las formas en que dichos dispositivos son producto de demandas, necesidades y requisitos de esos sectores para poder educarse en lugares que quieren y de la forma que prefieren así como para detentar y legitimar los puestos o cargos que en sus futuros ocuparan, en una época en la que el discurso de la meritocracia, de las competencias y del liderazgo se vuelve nodal para entender aquello que está sucediendo hoy en la sociedad.

Con estos sentidos, la interrogación gira en torno a qué particularidades presenta el dispositivo pedagógico de elite en las sociedades de gerenciamiento (Grinberg, 2008) en términos generales y también cabe preguntarse por las particularidades que presenta esa formación en liderazgo en esta institución en la actualidad. Para ello, aquí se retoman resultados de una investigación de grado denominada «La formación de liderazgo en tiempos de gerenciamiento. Un estudio de las prácticas institucionales y los sentidos de estudiantes en una escuela de elite del conurbano bonaerense ${ }^{1}$ que abarcó técnicas múltiples a partir del estudio en caso.

La estrategia metodológica utilizada fue múltiple y cualitativa a partir de la sistematización de fuentes documentales de la escuela, observación participante de los espacios institucionales así como de clases y la realización de entrevistas en profundidad con las autoridades y estudiantes del primero y el último año escolar. Ello se realizó en una institución de nivel secundario privado del conurbano bonaerense con orientación católica que enseña catequesis e infunde los valores del cristianismo aunque legalmente es laica y cuenta con esos servicios como actividades extraprogramáticas, donde los alumnos se reciben de bachilleres con orientación en ciencias sociales. Es un colegio que surge en los años '70 a través de 
un grupo de padres preocupados por fundar un lugar para sus hijos varones acorde con sus convicciones y donde se cultiven las virtudes humanas y las tradiciones.

Así, a continuación se realiza un breve repaso por algunas de las prácticas de formación de liderazgo que se configuran en el dispositivo pedagógico en esta institución atendiendo a algunas de las nociones teóricas y a las «prácticas discursivas» (Foucault, 2007a) que desde las lógicas de gerenciamiento se sostienen en la escuela, para luego adentrarnos en los sentidos de los estudiantes sobre sus elecciones futuras en correlación a sus procesos de escolaridad.

\section{Prácticas de formación de liderazgo y dispositivos pedagógicos de gerenciamiento}

Los estudios de Bowles y Gintis (1981) y de Baudelot y Establet (1990) desde el campo de la sociología de la educación, fueron los pioneros en describir y desarrollar los entramados, las dinámicas y los procesos de escolarización de forma separada y heterogénea de la población de clase burguesa y proletaria. Estos estudios fueron importantes para la problemática en cuestión en tanto que pusieron de manifiesto el doble circuito escolar diferenciado y segmentado. Por supuesto, en la Argentina estos desarrollos tuvieron su correlato en los estudios de Bravlasky (1985), Llomovatte (1991) y Tiramonti (2005) para comprender los procesos de segmentación y fragmentación educativa. Hacia la actualidad las brechas de la desigualdad en la educación del país se han profundizado y se produce, entre otras cosas, el estallido de esos procesos de fragmentación escolar (Guzmán, Langer y Grinberg, 2019). De allí la necesidad de producir investigaciones que describan estas realidades educativas actuales.

De hecho, si bien la formación conlleva históricamente valoraciones y proposiciones filosóficas en las maneras de hacer el dispositivo pedagógico (Grinberg, 2008; Langer, 2017; Armella, 2016), en el actual momento histórico de acumulación flexible (Harvey, 2000), las prácticas escolares en torno a los tiempos y espacios de la vida cotidiana escolar se van transformando. Ello sucede con la formación en liderazgo que se propone la institución a la que nos referimos, dado que es posible entenderla discursivamente y desde las prácticas del gerenciamiento (Grinberg, 2008) en la actualidad. Aquí, la noción de dispositivo pedagógico nos permite comprender los significados, normas, distribuciones temporales y espaciales que dan forma a la escolaridad en un tiempo y espacio histórico formando a los sujetos que transitan por dicha escolaridad (Grinberg, 2008). Es decir, es un dispositivo pedagógico que se produce ante las nuevas transformaciones que demandan del individuo mayor autonomía, flexibilidad y formación permanente, que procuran un nuevo tipo de subjetividad con carácter participativo, reflexivo, responsable y creativo, por mencionar algunas de las características principales a las que refieren algunos de los contenidos curriculares y de las dinámicas de la institución. En función de algunas de sus «prácticas discursivas» (Foucault, 2007a), es decir todo aquello que se hace pero también lo que está implícito a partir de las formulaciones filosóficas en documentos así como en las propuestas curriculares y actividades de la institución, los sujetos «deben» comprometerse y hacerse responsables de sí mismos. Desde estos puntos de vista, cambia radicalmente los términos de los procesos formativos en este espacio escolar. 
Con estas formas, las clases sociales dominantes buscan diferenciarse, más allá de los títulos, con escuelas propias que otorgan un círculo de socialización que haga las veces de «título de nobleza» (Gessaghi, 2016). Por ello, en la escuela a la que nos referimos, los jóvenes se vinculan con otros sujetos similares como una de las razones por las que las familias eligen dicha institución. Este tipo de instituciones procuran, siguiendo a Ziegler (2004), «constituirse como un pequeño mundo para los actores que las transitan.» (5). El tipo de formación que ofrecen es de carácter integral para ayudar al alumno a crecer como persona, fundada en los ideales de actores pertenecientes a una clase social alta que tienen la posibilidad, a la vez que la voluntad, de fundar sus propias escuelas. Ello con la finalidad de que sus hijos estén en una determinada red de relaciones y tengan la formación en valores tradicionales por un lado, a la vez que aquella que necesitan para desarrollarse en un mundo cada vez más competitivo, por otro lado. De hecho, desde la mirada de la conducción de la institución, la orientación en sociales se relaciona con que los futuros líderes «puedan observar el mundo social desde una mirada propia, capaces de reflexionar acerca de su propia existencia y poder actuar en consecuencia, verse capaces además de ser generadores del cambio social, innovar y emprender nuevos desafíos» (Director de la institución, Noviembre 2019).

Aquí, orientamos la mirada en un aspecto de este circuito segregado y que es, por supuesto, excluyente hacia otros que no pertenecen a esos sectores, a partir de la descripción de la escolarización, las experiencias educativas así como las prácticas pedagógicas, los procesos selectivos y los procesos de socialización de estudiantes que forman parte de los sectores más acomodados de la Argentina. Nos referimos a los estudiantes más favorecidos que, siguiendo el ya clásico estudio de Bourdieu y Passeron (1977), no deben sólo a su medio de origen hábitos, entrenamientos y actitudes que les sirven directamente en sus tareas académicas, heredan también saberes y un saber hacer, gustos cuya rentabilidad académica, aun siendo indirecta, no por eso resulta menos evidente. Sin duda, esas diferenciaciones según el origen social así como en relación a sus gustos, sus intereses, sus consumos proporcionan una óptica de las desigualdades sociales en las sociedades de gerenciamiento en la actualidad.

La formación de liderazgo que la institución propone apuesta por la lógica de las competencias en correspondencia con el modo de organización y gestión empresarial. Su implementación significa que tendencialmente es el individuo el que hace su puesto (Langer y Levy, 2009). Siguiendo a Grinberg (2008), ser competente involucra volverse una mercancía atractiva en el mercado de empleo y para ello es necesario que los sujetos desarrollen un conjunto de nuevas actitudes que, lejos de expresar algún tipo de diferencia originaria, son resultado de la construcción de las capacidades y cualidades que la escuela debe propiciar, potenciar y orientar para su consecución.

De esta manera, nos referimos a un conjunto de nuevas prácticas de la formación que delinean los «dispositivos pedagógicos del gerenciamiento» (Grinberg, 2014:201): 1. familias formando familias, 2. consejos de curso, 3. tutorías, 4. organización y participación en talleres y conferencias, 5. competencias de debate, 6. viajes al exterior. Son estas prácticas de la institución que generan los valores que todo líder de este mundo 
debe tener: autonomía, libertad, responsabilidad, competencias, habilidades proactivas, idiomas, posibilidad de debatir, polivalencia, trabajo en equipo.

Las tres primeras prácticas institucionales hacen referencia a las acciones sobre las familias, las guías que establecen con los estudiantes y las tutorías para que cada quien sea competente. Para ello es fundamental, desde el ideario de la institución, que los docentes generen un clima de confianza y motivación permanente, en el que su rol será el de guiar, dirigir, ser facilitadores, apoyar y transmitirles confianza en sí mismos a los alumnos para que se vean como emprendedores, comprometidos con su formación y capaces de tomar sus propias decisiones. En este sentido, asistimos a un movimiento que encorseta a la enseñanza en la función del coach (Grinberg, 2016), que debe orientar aprendizajes. El docente no solo debe transmitir conocimiento, debe además generar una cultura de vida, una cultura emprendedora, crea ambientes de aprendizaje autónomo, donde los alumnos tengan la posibilidad de aprender por sí mismos para luego, ser capaces de vivir el mundo e interpretarlo. Desde estas concepciones, el principal protagonista de la educación son los alumnos y la escuela debe acompañarlos y ayudarlos a ellos en esta tarea a través del desarrollo de distintos planes de mejora de su propia formación que incluyen prácticas distintas para cada edad.

Las tres últimas prácticas -los debates, la organización de conferencias y los viajes- ponen en valor aquello que «cada estudiante tiene» así como para que se responsabilice y se vea capaz de innovar, proponer ideas, ser competente. Estas características, son las de un líder en el mundo actual. Tal como lo dice uno de los objetivos del colegio, «aprender a emprender» se vuelve un eje central de la escolaridad, desplazando a un segundo plano al saber y los conocimientos. Ello se transforma en algo que los alumnos deben incorporar como prioridad. A partir de estas prácticas discursivas, los alumnos deben tener la suficiente apertura para permitirse pensar en ideas nuevas, confiar en ellos mismos y convertirse en fuentes de su propio crecimiento. Es decir, ser capaces de poner en juego tres aspectos fundamentales de las competencias tales como «el ser, ya que de él surge el interés y la motivación, el saber, con relación a los conocimientos que debe poseer y el saber hacer, que responde a las experiencias y habilidades» (Sierra Villamil, 2016:121). Desde las lógicas de estas prácticas discursivas, un alumno que puede manejar estas habilidades, estará dotado para transformarse en un líder, que con una actitud proactiva, las usará para tomar decisiones y elaborar sus propios proyectos, siendo además creativos, innovadores y capaces de lograr transformaciones. Así, es el estudiante que con la guía de un tutor va haciendo su propio camino, un «elige tu propia aventura» (Grinberg, 2008) que va configurando su escolaridad a medida, tal como se propone la formación para un sujeto que va a ocupar los puestos más alto del sector productivo. No se espera que le vayan delineando sus objetivos, sino que de la formación de un líder se espera que los configure él mismo. Como observa Grinberg (2014), en tiempos de gerenciamiento «formar a alguien, ahora, parece ser formarlo para que él mismo se forme, que se forme para buscar soluciones, e incluso se vuelvan responsables de su propia búsqueda» (204).

La escuela no sólo busca formar buenas personas que puedan ser «miembros responsables de una familia», sino además «ciudadanos comprometidos», «profesionales competentes» y «líderes capaces de innovar» (Ideario del colegio, Octubre 2019). Son estos valores y estos sentidos los que asumen en 
correspondencia, tal como veremos a continuación, los estudiantes de la escuela en relación a sus elecciones laborales futuras.

\section{Correspondencias con las elecciones laborales futuras de los estudiantes}

Los estudiantes de la escuela pertenecen, en muchos casos, a familias de lo que Roy Hora (2005) llama «burguesía terrateniente», ligadas históricamente a la sociedad rural, quienes supieron ser grandes terratenientes y productores, hoy devenidos en arrendatarios para la explotación de la soja. En otros casos, son los hijos de los socios de grandes bufetes de abogados, de los más prestigiosos del país, como así también de profesionales exitosos adaptados a la economía moderna. Son parte de una población que se recluye a barrios privados en busca de relaciones más cercanas y estilos de vida con gente que pertenece al mismo grupo, de los sectores más acomodados de la sociedad. Esta «segregación acallada o presuntamente indolente» (Carman, 2013:22) da cuenta del acceso a urbanizaciones en contacto directo con la naturaleza en grandes extensiones a un costo «relativamente bajo», así como por búsquedas de mayor «sensación de seguridad» dado que quieren una mejor vida, con menores riesgos y con mayores libertades. Quizás, son estos valores aquellos que sostienen principalmente las familias de los estudiantes así como muchas de las prácticas y los espacios que se corresponden con la institución a la que nos referimos.

Los sentidos que los estudiantes refieren, dan cuenta de ese tipo de vida al que apuestan, lejos de la ciudad, alejada del tránsito, de los riesgos, con espacios con mayor libertad y autonomía. Sin duda, la dimensión que se remarca en sus relatos es la libertad como parte central de esa socialización en la vida que luego se repite en la institución en torno a los espacios y tiempos, a las prácticas para crear sus propios planes de mejora en la formación, así como será crucial en sus vida futuras para tomar decisiones laborales. Esas libertades vienen asociadas a responsabilidades y toma de decisiones en la vida cotidiana.

Esa relegación socio espacial (Wacquant, 2007) es sostenida para el encuentro con semejantes. La segregación se produce desde lo espacial en los barrios, así como también hay una búsqueda de auto-segregación escolar que es la base y desde donde parten para pensar sus elecciones laborales futuras. Se eligen las familias y los compañeros de la escuela como forma de distinción de clase y también como resguardo de determinadas creencias. Tal como dice un estudiante, «tener las mismas formas de pensar así» como aquello que piensan sus familias. Estos sentidos expresan las posibilidades de construir un común a través de las formas de vivir, las formas de pensar, las maneras de educarse y, también, las relaciones para sus futuros laborales. La autosegregación escolar en las poblaciones de elite, sostenemos, se produce diferencialmente en búsqueda del sostenimiento de ciertos valores arraigados en sus tradiciones familiares y así el sistema educativo privado termina configurándose como un agregado institucional fragmentado (Tiramonti, 2005), donde lo prima en esta institución es una segregación por valores, tal como lo remarca otro alumno:

«Hasta donde sé, hasta todo lo que viví, yo lo viví acá adentro y con los valores que compartimos todos. Ese es otro tema, porque la gran mayoría está de acuerdo con las ideas del colegio a esta altura, en sexto año; capaz que en segundo tuvo un encuentro, 
a la familia no le terminó de cerrar, no está acá ya. Es una cuestión de que somos todos bastante parecidos y es eso, estamos todos en la misma línea». (Alumno de sexto año, 17 años, diciembre de 2019).

El estudiante hace referencia a compartir valores, ser bastantes parecidos y estar en la misma línea y al que no le cierran esos valores, tiene las puertas abiertas para irse tal como sucedió con un compañero. Así se configura este entramado de segregación escolar en función de valores, pensamientos, tipo de lenguaje y mentalidades acordes a las que esperan y tienen expectativas las familias que comparten los valores cristianos tradicionales y los futuros líderes de la sociedad. Estas construcciones de la «comunidad moral refuerza la idea de la opción por lo conocido, por aquello que no parece extraño ni amenazante ante los ojos de las familias más conservadoras, por aquello que promete el retorno a un pasado idealizado» (Tiramonti y Ziegler, 2008:50). Los alumnos tienen la idea de que sus familias pertenecen a un grupo de distinción en el que hay buenas personas pero que no son de cualquier familia y que más o menos se asemejan unos a otros, tienen las «mismas mentalidades».

En la elección escolar, las familias buscan garantizar una socialización entre pares homogéneos, signada por el mantenimiento de cierto status así como una inserción futura prometedora a través de los contactos necesarios para que los adolescentes puedan recrear las posiciones adquiridas por su familia. De esta manera, los estudiantes, siguiendo a Tiramonti y Ziegler (2008), entran en una red de relaciones que construye y mantiene su grupo social de pertenencia. Por otro lado, quizás como elemento central en estas elecciones, los estudiantes permanecen aislados de esas otras posibles socializaciones externas ya que fuera del colegio, tal como relata un alumno, no hacen nada. De hecho, la escuela se encarga también del tiempo libre de sus alumnos, ofreciendo espacios de participación extracurriculares planificadas como la escuelita de fútbol o el entrenamiento de rugby. La tradición escolar es que los jóvenes tengan actividad el fin de semana donde las familias concurren al campo de deportes ubicado en Pilar, cerrando aún más el circuito de socialización y diferenciación. Así, los jóvenes y sus familias interactúan con otros semejantes en los hogares, en la escuela y en actividades deportivas y culturales, así como sus encuentros y salidas nocturnas que las configuran en esa misma burbuja.

Sin embargo, también desde sus miradas tensionan y tratan de «romper» con su reclusión, con la segregación y la burbuja en la que están instalados hace tiempo en y desde sus posiciones de privilegio. Así, los estudiantes refieren a tratar de «salir para conocer el mundo». Allí hay una clave para entender la formación como líderes en tanto que les permite enfrentar lo que viene, ser competentes en el mundo «real», valerse y «hacerse a sí mismo» (Grinberg, 2008). Así se refieren a lo que viene, a su vida futura, en relación tanto a cuando terminen su escolaridad secundaria y a la continuación de su formación, así como a sus posibles salidas laborales. Aquí es donde los valores de la meritocracia y el esfuerzo se expresan con toda su intensidad: «yo estudié, yo me preparé para enfrentarme a este mundo». Por supuesto, estos valores tan instalados en nuestra sociedad, desde la institución a la que nos referimos y en la mirada de los estudiantes se desdibujan las situaciones de privilegio debido a su origen, con consonancias directas en la preparación para un mundo económico y laboral en cambio constante. Esa formación permanente e integral, en la figura de la educación superior, es vista 
por los estudiantes como un recurso más para el mundo que les viene. En muchos casos, los alumnos, no asistirán a universidades de confesión católica y esto, también, será una salida temporaria de la burbuja en el futuro, tal como dice un estudiante:

«El año que viene el que no estudia en la UCA eso no lo tiene más, viste. Entonces yo voy a estudiar en una universidad laica. La Di Tella que no es, no tiene nada de religión, de hecho todo lo contrario si querés» (Alumno de sexto año, 17 años, agosto de 2019).

Salir al mundo, desde este punto de vista, no es cambiar su vida radicalmente. Ello no implica asistir a una universidad pública, nacional y gratuita. En todo caso, es no continuar en la misma línea e ir a una universidad que tenga religión. Conocer el mundo y salir de la burbuja «puede entenderse exclusivamente como una estrategia de reproducción, dada la importancia de las redes sociales, ampliar el abanico de relaciones, aprender a convivir con los otros, etc., puede hacer la diferencia» (Gessaghi, 2016:94). Ir a una universidad privada, como la Di Tella, garantiza la posibilidad de ampliar ese mundo, el abanico de relaciones y redes a la vez que salirse de la burbuja de la religión y los valores exclusivamente tradicionales. Ello sin perder el status de ser parte de una selecta población que realiza estudios superiores y que lo hace en universidades pagas.

Salir de esa burbuja también implica desde sus sentidos, conservar lo que es necesario y lo que les da pertenencia al mismo tiempo que la necesidad de abrirse a otras cosas. Así, un estudiante hablaba de «todo lo que está pasando», todos los «quilombos que hay», esos ruidos tras los muros que el colegio no puede mantenerse ajeno así como la posibilidad de que frente a ello la escuela sea inflexible en «sacar chicos educados, respetuosos, muy comprometidos». Las familias y los alumnos buscan un colegio de elite que pueda traspasar sus muros para ofrecer una educación «más completa, con más mundo». Ya no es suficiente con una buena educación cultural y deportiva, basada en valores morales y tradicionales. Desde estos puntos de vista, se debe lograr flexibilidad para no quedar disociado de la realidad. De allí, la idea del estudiante de enfocarse en «sacar» personas además de educadas y respetuosas, también, muy comprometidas. La formación de líderes desde el punto de vista de este estudiante es formar una persona empapada de aquello que pasa en la sociedad. Así, un ejemplo de ello en relación a sus futuros laborales es la significación que otorgan al voluntariado y a la asistencia social que realizan en y desde la escuela. De este modo, un alumno habla sobre su futuro más allá de la universidad pensando acerca de una fundación en la cual le interesaría participar «si le dan los tiempos»:

\footnotetext{
«Es una fundación que está la mamá de un amigo, se encarga de ayudar a la gente a que progresen todos, no solo la niñez sino todos. Y capaz que el año que viene tenía tiempo arrancar con, ir a ayudar a eso» (Alumno de sexto año, 17 años, julio de 2019).
}

Ser voluntario es parte también de los discursos de las lógicas empresariales. Ayudar en todos los aspectos de la vida a través de fundaciones u ONG es aquello que permite a estudiantes de la educación superior con alto poder adquisitivo, ir adquiriendo experiencia y conociendo el mundo tal como es. De este modo, asistimos, como señalan Tiramonti y Ziegler (2008), «a una intensa exaltación 
del voluntariado entronizado con el descubrimiento de la «solidaridad privada», entendida como una virtud de la conciencia moral de cada persona» (125). Pero esta «vocación de servicio» no «viene dada» en los alumnos o en sus familias sino que es producto de que la escuela propone prácticas para ayudar a despertar esta «conciencia moral». Ser voluntario y ayudar en todos los aspectos de la vida a quien lo necesite implica esta vocación de servicio y la conciencia moral antes de «»entrar» directamente como trabajador-líder del sector productivo. A la vez que se estudia, hay un énfasis de los estudiantes de ir conociendo las realidades a través de estas actividades en fundaciones y ONGs.

A partir de estas experiencias, los jóvenes asocian trabajo a la vocación y a la realización personal. Así, al hablar sobre su propio futuro se ven ocupando lugares de prestigio y poder, alcanzando las máximas posiciones ya sean gerenciales en empresas, o, en menos casos, desde la dirigencia política. Tal como resaltaba el director, se produce un fenómeno inédito en estos jóvenes estudiantes que es la participación política y militancia a partir de la fundación del PRO en 2005. Con estos mismos sentidos, un alumno piensa en su futuro mediante el cambio que cree necesario y en cómo al empezar a estudiar historia y política, puede hacer algo desde y con la política:

«Quiero ser parte de eso, de las decisiones, digamos. Sí. Y tratar de llevar el país a ser mejor, a un mejor lugar. Poder ser un futuro dirigente (... prefiero hacer algo que quedarme pensando, quedarme sentado viendo qué hacen con la política o en la política.» (Alumno de primer año, 13 años, noviembre de 2019)

Como señala Vázquez (2020), «estos jóvenes pertenecientes a clases altas, con un perfil profesionalizado reconocen el trabajo en el Estado como una elección.» (6). El estudiante quiere ser parte de las decisiones, ser un futuro dirigente para tratar de mejorar al país. Por ello, la preocupación de sí se vuelve central en la sociedad de empresa (Foucault, 2007) y se vuelve una manera de vivir para todos y para toda la vida (Giraldo Díaz, 2008). Esto coincide con un cambio de discurso que, hasta hace poco era el dominante absoluto, acerca del descreimiento de la política como forma de participación. Es decir, es un cambio que a estos sujetos les permite ver al estado «como un ámbito de posible pertenencia cambiando percepciones y potenciando transformaciones.» (Vázquez 2020:13).

En estas perspectivas de los estudiantes sobre sus posibles inserciones laborales ya sea en términos políticos o empresariales, predomina el discurso de la gestión, tal como lo refiere explícitamente el siguiente estudiante:

«Podría estudiar economía, me encantaría tener los conocimientos y la cabeza y la
mentalidad pero lo mío sería más la gestión. Me parece, según lo que entendí de mí.
Es más la gestión, es más el trato, es más me gusta tener los conocimientos, me parece
una muy buena herramienta» (Alumnos de sexto año, 17 años, octubre de 2019).

Tanto en este relato como en el anterior, los jóvenes se perciben desde dos carreras bien diferentes, en cargos directivos y como futuros líderes, «gestionando», haciendo «trabajo técnico». La gestión es entonces la nueva forma de ejercer poder, inclusive más allá de los conocimientos, que para este alumno es «eso, una herramienta». Es decir, el discurso de la gestión, asumido por este estudiante pero también como veíamos en correspondencia por la escuela y las familias, hace referencia al mejoramiento de sus propias habilidades, 
capacidades y actitudes emprendedoras. Ser emprendedor, desde estas lógicas, es ser capaz de innovar, ser creativo y diferenciarse, poner en marcha propuestas y soluciones diferentes ante problemas o situaciones que puedan surgir, buscando activamente oportunidades de proyectos que generen un alto impacto. Son jóvenes que quieren ser líderes y exitosos y para ello buscan ejemplos, como los casos de emprendedores que escuchan en conferencias y los llaman para aprender de ellos. Buscan conocer ese mundo, incentivados por las prácticas escolares, organizando actividades que corresponden a la vida que quieren elegir. Es decir, apuestan a volver a la burbuja. Para ello, los conocimientos y saberes le son necesarios e imprescindibles, más allá de las carreras que sean. Estos jóvenes no se plantean la posibilidad de no realizar una carrera universitaria. La educación y la adquisición de conocimientos y saberes desde el punto de vista de estos estudiantes están directamente ligadas al tipo de empleo que tendrán así como al nivel de éxito económico en sus futuros.

Sin duda, estos discursos están ligados a aquellas teorías del capital humano (Schultz, 1972) ahora devenidas en discursos del management y de la gestión (Grinberg, 2014) que se transmiten y reproducen tanto en sus familias como en las prácticas cotidianas de la escuela. Es en esas prácticas del colegio que hay una búsqueda para que los estudiantes alcancen su mejor versión, ser exigentes, ser perfectos, ser líderes, ser competentes. Así hablan los estudiantes de quienes quieren ser y, además, cómo el colegio afecta a esas posibilidades a través del lenguaje de la gestión en torno a «la excelencia, la calidad total, el logro de cero defecto, los círculos de calidad, la planificación por proyecto, la autonomía, el liderazgo, la innovación» (Grinberg, 2014:104) que constituyen los aspectos centrales de la gestión como episteme.

\section{Reflexiones finales}

La formación de liderazgos en esta escuela de elite se abordó a partir de las preguntas por las prácticas institucionales, así como por quiénes son los estudiantes y quiénes quieren ser. Por ello, la preocupación fue sobre la producción de las subjetividades en la sociedad de empresa (Foucault, 2007) y de gerenciamiento (Grinberg, 2008) a través de la descripción de los valores, las prácticas y los sentidos que se cierran en un círculo propio de segregación en el que la escuela prepara y forma para el managment, el emprendedurismo y el liderazgo en un marco de acumulación flexible (Harvey, 2000) en el que no hay lugar para todos (Castel, 1995).

Así, dimos cuenta de las formas en que ciertas prácticas institucionales forman sujetos para un futuro en el que deben pensarse como líderes, responsables, autónomos, con habilidades que den cuenta, siguiendo a Sennett (2006), de sus posibilidades de pensarse como trabajadores flexibles, capaces de adaptarse y pasar de un trabajo a otro sin demasiado tiempo para detenerse, trabajar en equipo, aceptar la evaluación permanentemente y constituirse en los más aptos, eficientes, eficaces, gestores y competitivos. Allí nos detuvimos en describir las correspondencias de esas prácticas con los sentidos que los jóvenes construyen en relación a sus elecciones laborales.

Esos sentidos sobre sus futuros, sus expectativas personales y laborales así como los proyectos que producen los jóvenes desde esta experiencia escolar, aunque no 
solamente porque también provienen y fueron «afectados» por sus pertenencias de clase de elite, implica un tipo de formación recibida en la institución en torno a discursos y prácticas del hacerse a sí mismo, del emprendedurismo, de la competencia, de la inmersión, autonomía, independencia, libertad y posibilidades que demuestran y expresan a partir de sus deseos por convertirse en futuros líderes, sea en los espacios y lugares de educación superior o laborales que sean. Sin duda, con estos mecanismos de realización es donde el sistema capitalista funciona con toda su plenitud y esplendor, pero también tal como dice Harvey (2000), «aun cuando la capacidad aparentemente infinita para generar productos alimente todas las ilusiones de libertad y caminos para la realización personal, el capitalismo lleva consigo su aparto ilusorio, sus fetichismos y su sistema de espejos allí donde va.» (377).

Esas múltiples decisiones y elecciones que realizan cotidianamente los jóvenes tanto en sus formas de vivir, en sus procesos de formación así como hacia sus vidas laborales son configuradas y producidas a través de un novedoso dispositivo pedagógico desde el que sostienen los valores del gerenciamiento (Grinberg, 2008) y desde el que proponen prácticas, actividades y procesos en los que la competitividad, autonomía, responsabilidad, gestión, libertad, emprendedurismo, innovación, adaptación, son solo algunos de los rasgos centrales que caracterizamos en la escuela de elite y que los estudiantes sostienen, defienden y legitiman con formas que se retraolimentan y corresponden con esas prácticas discursivas.

Por tanto, el objetivo que orientó la búsqueda de este trabajo se basó por un lado en conocer y reconstruir los procesos de socialización y escolarización de jóvenes que asisten a una escuela de élite visibilizando la relación que se produce entre la educación y el acceso a posiciones de privilegio y poder a través de un tipo de formación que apuesta a la producción de liderazgos. Son estos sectores más poderosos que buscan caminos de diferenciación para evitar el riesgo de un sistema que los iguale al resto de la población asegurándose que sus hijos asistan a instituciones con determinados patrones culturales, valores, características económicas similares, de la misma escala social, es decir una socialización entre «semejantes». Por otro lado, se trató de enfatizar esas correspondencias entre las prácticas discursivas institucionales y aquellas que sostienen los jóvenes en relación a sus escolaridades, a la continuación de sus estudios superiores así como sus elecciones laborales futuras. Es quizás allí donde observamos la eficacia de este tipo de dispositivos para producir sujetos que legitiman el proceso de éxito del cual ya forman parte a la vez que se convierten en funcionales a las lógicas del capitalismo actual.

Estas prácticas y estos sentidos configuran un determinado tipo de dispositivo pedagógico de gerenciamiento desde el cual no solo se legitima la desigualdad sino que se sostiene una determinada población como elite en torno a argumentos de competencias y méritos desde los que solo permanecen y llegan algunos pocos. Tal como señala Sennett (2006), es así y con estas formas que se producen discursos y juicios «enormemente personalizados» que determinan quiénes deben permanecer. Pero, también, decretan quiénes quedan fuera. 


\section{Referencias}

Armella, J. (2016). "Conectar igualdad: o la irrupción de las netbooks en la escuela”. Trama de la Comunicación. Rosario, V. 20, n. 1, pp. 197-215.

Baudelot, C. y Establet, R. (1990). La escuela capitalista. Bs. As: Siglo Veintiuno Editores.

Bourdieu, P. y Passeron, J. (1977). La reproducción. Barcelona: Laia.

Braslavsky, C. (1985). La discriminación Educativa en Argentina. Bs. Aires: Grupo Editor Latinoamericano.

Carman, M., Neiva V. y Segura, R. (2013.) "Introducción. Antropología, diferencia y segregación urbana.” En M. Carman, V. Neiva \& R. Segura (coords.) Segregación $y$ diferencia en la ciudad (pp. 11-34). Quito: Flacso-Ecuador; Clacso.

Foucault, M. (2007). Nacimiento de la biopolitica, Bs. As.: Fondo de Cultura Económica.

Foucault, M. (2007a). La arqueología del Saber. Buenos Aires: Siglo Veintiuno editores.

Gessaghi, V. (2016). La educación de la clase alta argentina. Buenos Aires: Siglo Veintiuno Editores.

Giraldo Díaz, R. (2008). La resistencia y la estética de la existencia en Michel Foucault. Entramado, pp. 90-100.

Grinberg, S. (2008) Educación y poder en el siglo XXI. Gubernamentalidad y Pedagogía en las sociedades de gerenciamiento: Buenos Aires.

Grinberg, s. (2014) "De la disciplina al gerenciamiento, del examen al monitoreo. Un estudio sobre el gobierno y la evaluación en las sociedades contemporáneas". En Revista de la Asociación de Sociología de la Educación N² 2 pp. 155-172.

Grinberg, S. (2016) "Elogio de la transmisión. La escolaridad más allá de las sociedades de aprendizaje". En Revista Polifonias $\mathrm{N}^{\circ}$ 8. pp. 71-94

Guzmán, M., Langer, E. y Grinberg, S. (2019). Educación, trabajo y redes de escolarización en el capitalismo flexible: Un estudio en localidades del Golfo San Jorge. En Revista Trabajo y Sociedad, № 35, Vol XXI, pp. 603-623.

Harvey, D. (2000). Las condiciones de la posmodernidad. Bs. As.: Amorrortu.

Langer, E. (2017). Escuela, pobreza y resistencia. Defensas y luchas cotidianas de estudiantes. Rada Tilly: Del Gato Gris.

Langer, E. y Levy, E. (2009). "Educación de jóvenes y adultos, política social y gubernamentalidades en el siglo XXI". En Revista de la Escuela de Ciencias de la Educación. Facultad de Humanidades y Artes. Universidad Nacional de Rosario. Año 5.pp. 229- 250

Llomovatte, S. (1991). Adolescentes entre la Escuelay el trabajo. Bs. As.: FLACSO / Miño y Dávila ed.

Rose, N. (1996). “ $¿$ The death of the social? Re-figuring the territory of government”. En Economy and Society 25. Routledge: London. pp. 327-356.

Roy Hora (2005). La burguesía terrateniente: Argentina, 1810-1945. Texas: Capital Intelectual.

Schultz, T. W. (1972). "Inversión en Capital Humano". En Blaug M.: Economía de la Educación. Textos escogidos. Tecnos. Madrid: Siglo XXI, pp. 13-33.

Sennett, R. (2006). La cultura del Nuevo capitalismo. Barcelona: Anagrama.

Sierra Villamil, G. (2016) "Liderazgo educativo en el siglo XXI, desde la perspectiva del emprendimiento sostenible”. En Revista EAN N ${ }^{\circ}$ 81. (P. 111-128).

Tiramonti, G. (2005). La nueva configuración fragmentada del sistema educativo. Buenos Aires: Mimeo/ FLACSO. 
Tiramonti, G., Ziegler S. (2008). La educación de las elites. Aspiraciones estrategias y oportunidades. Buenos Aires: Paidós.

Vázquez, M. (2020) "Reconfiguraciones de las élites estatales y de las producciones socioestatales de juventudes en Argentina (2015-2019)". En Perfiles Latinoaméricanos $\mathrm{N}^{\circ} 55$. México: FLACSO.

Wacquant, L. (2007). Los condenados de la ciudad. Gueto, periferia y Estado. Buenos Aires: Siglo XXI.

Ziegler, S. (2004). Los de excepción: Un retrato de las elecciones escolares de las familias de sectores favorecidos en la Argentina. Buenos Aires: FLACSO.

\section{Notas}

1 Tesina de grado para la carrera Lic. en Educación de la UNSAM.

\section{Notas de autor}

Sobre el autor

Profesor en EGB 1 y 2. Vicedirector de escuela primaria. Referente de literatura infantil y juvenil Estudiante de la Lic. en Educación en UNSAM próximo a graduarse.

Sobre el autor

Post Doctor (Universidad Nacional de San Martín), Doctor en Educación (Universidad de Buenos Aires), Magister en Ciencias Sociales con mención en Educación (FLACSO/Argentina) y Licenciado en Ciencias de la Educación (Universidad de Buenos Aires). Es Investigador Adjunto del Consejo Nacional de Investigaciones Científicas y Técnicas (CONICET), es Director de las carreras de Educación en la Universidad Nacional de San Martín y se desempeña como Profesor de Sociología de la Educación por la Universidad Nacional de General San Martín y la Universidad Nacional de la Patagonia Austral. 